\title{
STUDY OF BIOFILM FORMATION AS A VIRULENCE MARKER IN CANDIDA SPECIES ISOLATED FROM VARIOUS CLINICAL SPECIMENS.
}

Saroj Golia, Vivek Hittinahalli, Sangeetha K. T, Vasudha C. L.

\author{
1. Professor \& HOD, Department of Microbiology, Dr. B. R. Ambedkar Medical College. \\ 2. Associate Professor, Department of Microbiology, Dr. B. R. Ambedkar Medical College. \\ 3. Post Graduates Student, Department of Microbiology, Dr. B. R. Ambedkar Medical College. \\ 4. Post Graduates Student, Department of Microbiology, Dr. B. R. Ambedkar Medical College.
}

\section{CORRESPONDING ADDRESS:}

Dr. Vivek Hittinahalli, NABH Assessor

Associate Professor, Dr B.R Ambedkar Medical College

E-Mail: vivekhitt@yahoo.com,

Phone: 00917760984581.

ABSTRACT: BACKGROUND: Candida species can be either commensals or opportunistic pathogens with the ability to cause a variety of infections, ranging from superficial to life threatening. Nosocomial infections due to candida are also becoming increasingly important. Early and prompt diagnosis, proper treatment and prevention of candidemia due to biofilms pose a major challenge for microbiologists and clinicians worldwide. Added to this is the emerging trend of antifungal drug resistance among the biofilm producing strains of Candida. AIMS: The aim of this study was to detect biofilm production in Candida species isolated from various clinical samples obtained from patients hospitalized in Dr. B.R Ambedkar Medical College and Hospital. MATERIALS AND METHODS: A total of 108 Candida species (Candida albicans49 and non-albicans Candida59 species) isolated from various specimens (urine, blood, respiratory tract, genital samples, plastic devices and pus samples) were included in the study.The various candida isolates were identified by using conventional methods and their ability to produce biofilm was detected by the tube method. RESULTS: Out of 108 candida species, non-albicans Candida 59(54.63\%) was the predominant species isolated. Biofilm positivity was seen with $71(65.74 \%)$ isolates and the biofilm production was observed more with non-albicans Candida species 44(61.97\%) compared to C.albicans species $27(38.03 \%)$. Among the non-albicans Candida species, strong biofilm producers were C.krusei(80.77\%) and C.tropicalis(72.73\%). Biofilm positivity was found to be higher in the bloodstream Candida isolates $(81.82 \%)$ compared to isolates from other sites. CONCLUSION: The present study suggests an increasing prevalence of non-albicans Candida species in the various clinical samples isolated and also shows them as strong biofilm producers compared to C.albicans species. These data suggest that, biofilm formation as a potential virulence factor might have a higher significance for non-albicans Candida species than for C.albicans and also that the biofilm structure varies with the different species and strains of candida, the nature of the colonized surface and its localization. Thus more remains to be determined about biofilms formed by the non-albicans Candida species as they are now frequently encountered species in catheter associated candidaemias.

KEY WORDS: Candida, biofilm, non-albicans Candida, candidaemia 
INTRODUCTION: Pathogenic fungi in the genus Candida are capable of causing a variety of infections ranging from superficial to deep-seated mycoses. The Candida species have been recognized as the fourth commonest cause of nosocomial invasive infections. ${ }^{1}$

Candida organisms are commensals; and to act as pathogens, interruption of normal host defences is necessary. Therefore, general risk factors for Candida infections include immune-compromised states, diabetes mellitus, and iatrogenic factors like antibiotic use, indwelling devices, intravenous drug use, and hyperalimentation fluids. Candidiasis has emerged as an alarming opportunistic disease as there is an increase in number of patients who are immune-compromised, aged, receiving prolonged antibacterial and aggressive cancer chemotherapy or undergoing invasive surgical procedures and organ transplantation. The virulence factors expressed by candida species, to cause infections may vary depending on the type of infection, the site and stage of infection and the nature of the host response. ${ }^{2}$ One of the important factors contributing to the virulence of candida is the formation of surface-attached microbial communities known as "biofilm". ${ }^{3}$

Biofilms are defined as structured microbial communities that are attached to a surface and encased in a matrix of exopolymeric material. A typical laboratory fungal model of biofilm formation involves two operational steps: (a) adhesion and (b) biofilm growth and maturation and has 3 distinct developmental phases: early $(0-11 \mathrm{~h})$, intermediate (12-30 h) and mature (38-72 h). The detailed structure of mature C.albicans biofilms consists of a dense network of yeast, hyphae and pseudohyphae. ${ }^{4}$

The advantages of forming biofilm include protection from the environment, nutrient availability, metabolic cooperation and acquisition of new traits. ${ }^{2}$ This is of particular significance since it is now estimated that a significant proportion of all human microbial infections involve biofilm formation. It has been estimated that some $65 \%$ of all human microbial infections involve biofilms. Biofilm formation helps the organism to evade host defences, exist as a persistent source of infection and develop resistance against antifungal agents. Candida species are frequently found in the normal microbiota of humans, which facilitates their encounter with most implanted biomaterials and host surfaces. ${ }^{5}$ The resistance of biofilm producing Candida species to antifungal agents represents a major challenge especially in the design of therapeutic and prophylactic strategies. These factors constitute a clinical problem, resulting in high mortality as well as economic problem due to prolonged hospital stay. ${ }^{6}$

The role of bacterial biofilms in disease has been investigated in detail over a number of years and considerable literature is available on their structure and properties. However, sufficient literature is hard to find on medically relevant fungal biofilms particularly, in the prevailing scenario where immune-compromised conditions and nosocomial infections are on the rise. Consequently further recognition and understanding of candida biofilms, is of major importance in the study of human candidiasis. Therefore this study aims to provide insights on various aspects of Candida biofilms and their role in pathogenesis.

MATERIALS AND METHODS: In this study, a total of 108 clinical isolates of Candida were collected from patients hospitalized in Dr. B.R Ambedkar Medical College and Hospital during a period of 6 months i.e. from May 2012 to October2012. These patients had no history of antifungal drug exposure prior to collection of sample.

Out of the 108 Candida isolates, 34 were obtained from urine,27 from respiratory tract(sputum, bronchial wash, tracheal secretion), 22 from blood, 16 from genital samples, 6 
from plastic devices (endotracheal tube, catheter tip) and 3 from pus samples[Table 1].The urine isolates were obtained from catheterised patients with symptoms of urinary tract infections, the respiratory samples were from pulmonary tuberculosis cases and the bloodstream isolates were collected from ICU patients with catheter related septicemias. The genital samples were collected from cases with vulvovaginal candidiasis. The plastic devices were from ICU patients and the pus samples were laboratory isolates.

The candida isolates obtained were further identified by conventional methods such as germ tube test [figure 1], microscopic morphology on cornmeal agar [Figure 2] and sugar fermentation and assimilation tests. ${ }^{7}$ Culture on CHROM agar was also used for identification of the species [Figure 3].

BIOFILM FORMATION: Biofilm production was detected by tube method described by Brachiniet al. ${ }^{8} \mathrm{~A}$ loopful of organisms from Sabouraud's Dextrose agar(SDA) plate was inoculated into Sabouraud's Dextrose broth supplemented with glucose (Final concentration $8 \%$ ). The tubes were then incubated at $37^{\circ} \mathrm{C}$ for 24 hours after which the broth was aspirated out gently. The tubes were then washed once with distilled water and then stained with $1 \%$ Safranin. The tubes were then kept still for 7 minutes. Safranin then was removed and tubes were examined for biofilm production. Biofilm production was tested twice and read independently by two different observers. The adherent biofilm layer was scored visually as either negative or weak positive (1+), moderate positive $(2+)$ or strong positive $(3+)$ [Figure 4].

STATISTICAL ANALYSIS: The analysed results were expressed as percentages for the description of candida isolates according to species and various clinical samples. Microsoft excel was used for the interpretation of these results.

RESULTS: Among the 108Candida isolates, 59 (54.63\%)were non- albicans Candida species and 49(47.37\%) were Candida albicans (C.albicans).Among the non- albicans Candida species, the most common isolate was C.krusei $26(24.07 \%)$ followed by C.tropicalis $11(10.19 \%)$. Other species isolated were C.glabrata $8(7.41 \%)$, C.kefyr $7(6.48 \%)$, C.parapsilosis $4(3.7 \%)$ and C.dubliniensis 3(2.78\%).[Chart 1]

The Candida isolates obtained from different clinical samples are shown in table 1.

Out of 108 candida species tested 71(65.74\%) were found to be biofilm producers. Biofilm production was found to occur most frequently among non-albicans Candida44 $(61.97 \%)$ than Candida albicans 27(38.03\%).

Among the non-albicans Candida species, C. krusei23 (80.77\%) was the highest biofilm producer followed by C.tropicalis 8(72.72\%).[Table 2 and Chart 2].

The results of biofilm production were also analysed with respect to the site of infection. The biofilm positivity was observed more with bloodstream isolates (81.82\%) and least with isolates from respiratory tract (40.74\%) [Table 3 and Chart 3].

DISCUSSION: The incidence of nosocomial candidiasis has increased dramatically over the last few decades. Their emergence as important nosocomial pathogens is related to specific risk factors associated with modern medical procedures, notably the use of immunosuppressive and cytotoxic drugs, powerful antibiotics, and implanted devices of various kinds. ${ }^{1}$ 
Biofilms are a collection of microorganisms surrounded by the slime they secrete. The ability to form biofilms is associated with the pathogenicity and as such should be considered as an important virulence determinant during candidiasis. ${ }^{9}$

In our study we evaluated 108 candida species from various clinical samples, namely urine, blood, respiratory tract, genital samples, plastic devices and pus. Our data showed predominance of non-albicans Candida species 59(54.63\%) compared to C.albicans49(45.37\%).Studies by Mujikaet al ${ }^{10}$ and Shin et al ${ }^{11}$ also indicate a trend towards an increasing prevalence of infections caused by species of non-albicans Candida. In the present study $65.74 \%$ of the Candida isolates tested were found to be biofilm producers. This finding is in concordance with studies conducted by Muni et al., 2012(64\% ${ }^{9}$ and Mohandas et al., $2011(73 \%)^{2}$.Biofilm production was found to occur most frequently among Non-albicans Candida species (61.97\%) than C.albicans(38.03\%). Similar findings have been reported by Girishet al., 200612and Muni et al., 201299). Among the non-albicans Candida species, the biofilm positivity occurred most frequently among isolates of C.krusei (80.77\%), followed by C.tropicalis (72.73\%), C.dubliensis (66.66\%), C.kefyr (57.14\%), and C.parapsilosis (50\%). [Table2 and chart 2]. C.krusei and C.tropicalis have also been recognized as strong slime producers by many studies (Dag et al., 201013; Mohandas et al., $2011^{2}$ and Vinithaet al.,200714).

Our study also showed correlation between biofilm productions by the various Candida isolates with respect to their source of isolation. Nearly $81.82 \%$ of the bloodstream isolates showed higher biofilm positivity(studies by Girishet al., ${ }^{12}$ also showed similar results), followed by urine isolates (73.53\%), genital samples (68.75\%),pus and plastic devices(66.67\%) and respiratory tract samples(40.74\%)[Table 3 and chart 3].The bloodstream isolates were collected from ICU patients with catheter related septicemias. Most of these infections are caused by microorganisms that invade the intracutaneous wound during catheter insertion or thereafter. ${ }^{15}$ The cultures from blood samples yielded C.krusei predominantly. The isolation of non-albicans Candida species in catheter associated bloodstream infections have been frequently encountered in the past few decades. ${ }^{16}$ The urine isolates were obtained from catheterised patients with symptoms of urinary tract infections and the cultures yielded mainly C.albicans. The Candida species isolated from plastic devices (endotracheal tube, catheter tip) also showed $66.67 \%$ of biofilm positivity. These devices become colonized by the candida that forms biofilm, the detachment of which can result in candidemia. Indwelling catheters therefore, represent a major risk factor associated with nosocomial Candida infections. ${ }^{14}$ Devices such as stents, shunts, prostheses, implants, endotracheal tubes, pacemakers, and various types of catheters, to name a few, have all been shown to support colonization and biofilm formation by Candida. ${ }^{5}$ The least biofilm producers were the isolates from respiratory tract $(40.74 \%)$ which were obtained from pulmonary tuberculosis cases and these results were in concordance with studies done by Muni et al., 2012. ${ }^{9}$

Candida biofilms may help maintain the role of fungi as commensals and pathogen, by evading host immune mechanisms, resisting antifungal treatment, and withstanding the competitive pressure from other organisms. Consequently, biofilm related infections are difficult to treat. ${ }^{17}$ Hence the study emphasizes the need for an effective anti-biofilm treatment which requires improved knowledge of the pathogen itself, and also of the host response to adhesion and biofilm formation, the properties of the substrates onto which the biofilm develop and the interactions within microbial communities.

To conclude, biofilm formation as a virulence factor might have a higher significance for non- albicans Candida species than for C. albicans and also this ability to form biofilms is 
intricately linked with the ability of the organisms to adhere, colonize and subsequently cause infection in susceptible individuals.

\section{REFERENCES:}

1. Douglas LJ (2003). Candida biofilms and their role in infection. Trends Microbiol., 11: 30-36.

2. Mohandas V, Ballal M. Distribution of Candida Species in Different Clinical Samples andTheir Virulence: Biofilm Formation, Proteinase and Phospholipase Production: A Study on Hospitalized Patients in Southern India.J Glob Infect Dis. 2011; Jan-Mar; 3(1): 4-8.

3. SeneviratneCJ, Jin L, Samaranayeke LP. Biofilm lifestyle of Candida: A mini review Oral Dis. 2008; 14: 582-590.

4. Aparna MS, Yadav S. Biofilms: Microbes and Disease. The Braz.J. Infect. Dis.2008; 12: 526-530.

5. RM Dominic, S Shenoy, S Baliga .Candida biofilms in medical devices: Evolving trends. Kath Univ Medical J. 2007; Vol. 5, No. 3, Issue 19, 431-436.

6. GILBERT, P. [et al.], ed. lit. - "Bolfilms : coming of age”. Biofilm Club : Manchester, 2007. ISBN 0-9551030-1-0. p. 33-41.

7. Larone, DH, Medically Important Fungi: a Guideto Identification. 1979. 2nd ed. Harper and Row Publisher, Hager's town. Maryland, New York,San Francisco, London.

8. Branchini, M. L., M. A. Pfaller, J. Rhine-Chalberg, T. Frempong, and H. D. Isenberg. Genotypic variation and slime production among blood and catheter isolates of Candida parapsilosis. J. Clin. Microbiol .1994; 32:452-456.

9. Muni S, Menon S, Chande C, Gohil A, Chowdhary A, Joshi A. Candida biofilm. Bombay Hosp J. 2012; Vol. 54, No. 1.

10. 10. Mujika MT, Finquelievich JL, Jewtuchowicz V, Iovannitti CA. Prevalence of Candida albicans and Candida non-albicans in clinical samples during 1999-2001. Rev Argent Microbiol. 2004; 36:107-12.

11. 11. Shin JH, Kee SJ, Shin MG, Kim SH, Shin DH, Lee SK, Suh SP, RyangDW. Biofilm production by isolates of Candida species recovered from nonneutropenic patients: comparison of bloodstream isolates with isolates from other sources. J. Clin. Microbiol.2002; 40: 1244-1248.

12. Girish Kumar, CP and Menon, T. Biofilm production by clinical isolates of Candidaspecies.Medical Mycology 2006; 44:99-101.13.

13. Dag I, Kiraz N, OZ Yasemin. Evaluation of different detection methods of biofilm formation in clinical Candida isolates. African J of Microbial Research.2010; Vol. 4(24), pp.2763-2768.

14. Vinitha, M and Ballal, M. Biofilm as virulence marker in Candida isolated from blood, World Jfor Medical Sciences 2007;2:46-48.

15. Chakrabarti A, Singh K, Das S. Changing face of nosocomial candidaemia. Indian J Med Microbiol.1999; 17:160-6.

16. Segal, E. and D. Elad. Candida species and Blastoschizomycescapitatus. In: Agillo, L. and R.J.Hay (Eds).1998; Microbiology and Microbial Infection. New York, Arnold, pp: 423460. 
17. Baillie GS, Douglas LJ. Candida biofilm and their susceptibility to antifungal agents.Methods Enzymol.1999; 310:644-56.

Table 1: Candida species isolated from different clinical samples $(n=108)$

\begin{tabular}{|l|l|l|l|l|l|l|}
\hline $\begin{array}{l}\text { No. of candida } \\
\text { species isolated }\end{array}$ & Urine & Respiratory tract & $\begin{array}{l}\text { Bloo } \\
\text { d }\end{array}$ & $\begin{array}{l}\text { Genital } \\
\text { samples }\end{array}$ & $\begin{array}{l}\text { Plastic } \\
\text { devices }\end{array}$ & Pus \\
\hline C.albicans(49) & 16 & 12 & 5 & 12 & 2 & 2 \\
\hline C.krusei (26) & 5 & 9 & 8 & 1 & 2 & 1 \\
\hline C.tropicalis(11) & 3 & 4 & 3 & 0 & 1 & 0 \\
\hline C.glabrata(8) & 6 & 0 & 1 & 1 & 0 & 0 \\
\hline C.kefyr (7) & 3 & 0 & 1 & 2 & 1 & 0 \\
\hline C.parapsilosis(4) & 1 & 0 & 3 & 0 & 0 & 0 \\
\hline C.dubliniensis(3) & 0 & 2 & 1 & 0 & 0 & 0 \\
\hline Total (108) & 34 & 27 & 22 & 16 & 6 & 3 \\
\hline
\end{tabular}

Table 2: Biofilm production by various Candida species

\begin{tabular}{|l|l|l|l|l|l|l|}
\hline Candida spp. & Total no. of & No. of biofilm & \multicolumn{3}{|l|}{ No. of biofilm } & \multicolumn{2}{l}{ Total no. of biofilm } \\
positive
\end{tabular}

Table 3: Biofilm production in various clinical samples

\begin{tabular}{|c|c|c|c|c|c|c|}
\hline \multirow[t]{2}{*}{ Nature of specimen } & \multirow[t]{2}{*}{$\begin{array}{l}\text { Total no. of } \\
\text { isolates }\end{array}$} & \multirow[t]{2}{*}{$\begin{array}{l}\text { No. of biofilm } \\
\text { negative }\end{array}$} & \multicolumn{3}{|c|}{$\begin{array}{l}\text { No of biofilm } \\
\text { positive }\end{array}$} & \multirow[t]{2}{*}{$\begin{array}{l}\text { Total no. of biofilm } \\
\text { positive }\end{array}$} \\
\hline & & & $3+$ & $2+$ & $1+$ & \\
\hline Urine & $34(31.48 \%)$ & $9(26.47 \%)$ & 6 & 10 & 9 & $25(73.53 \%)$ \\
\hline Respiratory tract & $27(25 \%)$ & $16(59.26 \%)$ & 2 & 7 & 2 & 11(40.74\%) \\
\hline Blood & $22(20.37 \%)$ & $4(18.18 \%)$ & 3 & 5 & 10 & $18(81.82 \%)$ \\
\hline Genital samples & $16(14.81 \%)$ & $5(31.25 \%)$ & 1 & 5 & 5 & $11(68.75 \%)$ \\
\hline Plastic devices & $6(5.55 \%)$ & $2(33.33 \%)$ & 2 & 1 & 1 & $4(66.67 \%)$ \\
\hline Pus & $3(2.78 \%)$ & $1(33.33 \%)$ & 1 & 1 & 0 & $2(66.67 \%)$ \\
\hline Total & 108 & $37(34.26 \%)$ & 15 & 29 & 27 & 71(65.74\%) \\
\hline
\end{tabular}




\section{ORIGINAL ARTICLE}

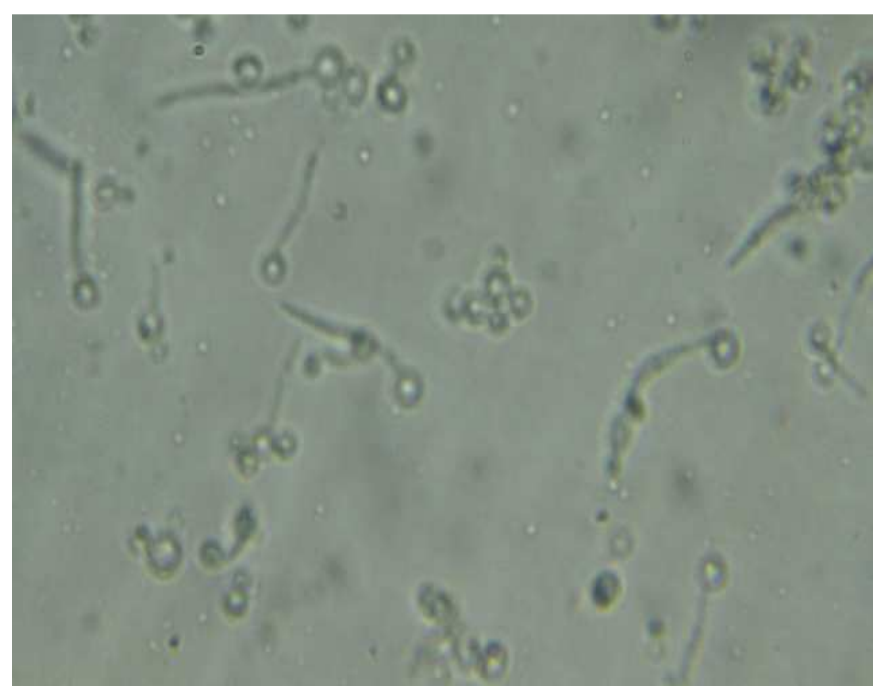

Figure 1.Germ tube formation by C.albicans

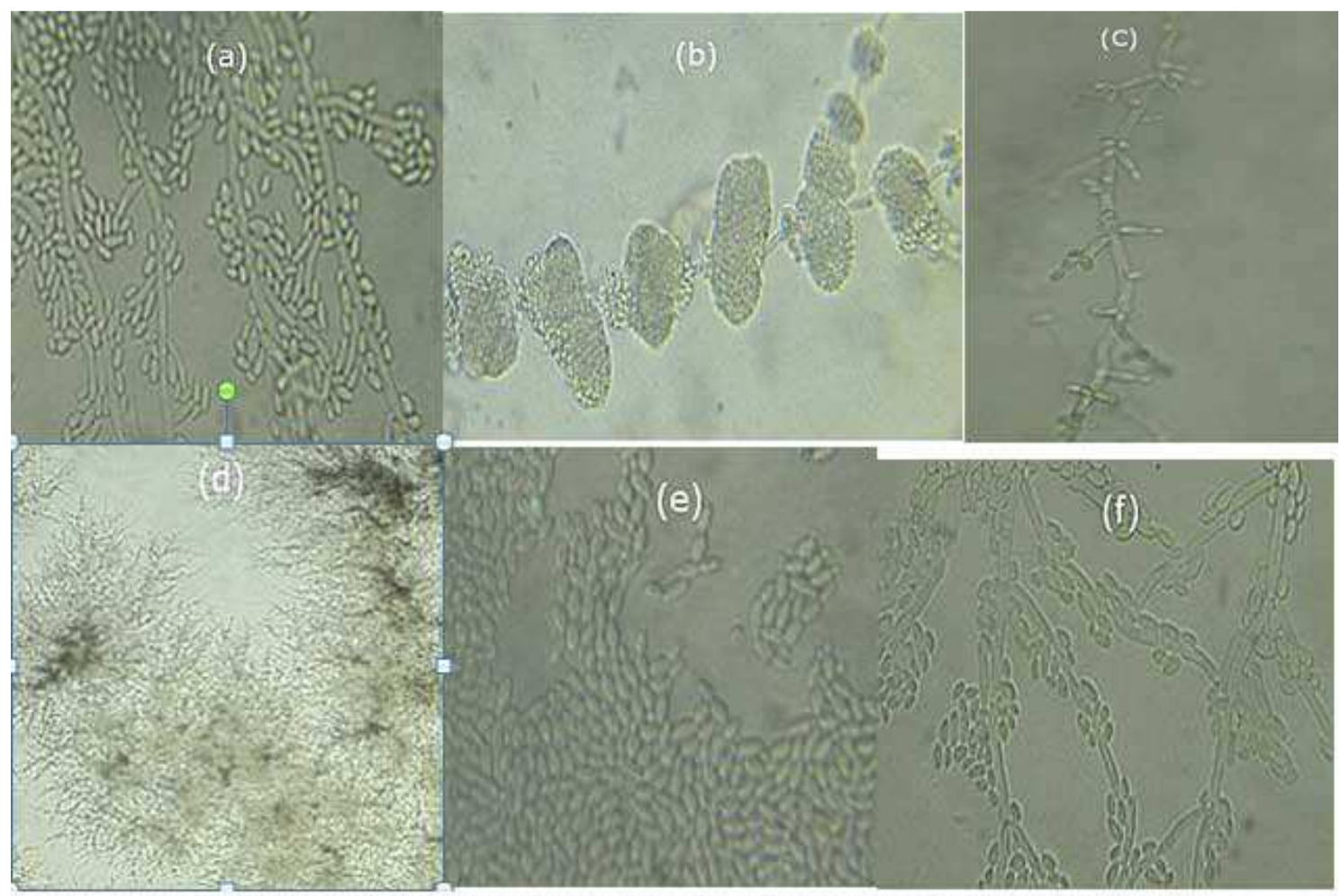

Figure 2. Microscopic morphology on cornmeal agar:C.krusei (a), C.albicans (b), C.tropicalis(c),C.parapsilosis(d), C.glabrata(e) and C.kefyr (f). 


\section{ORIGINAL ARTICLE}

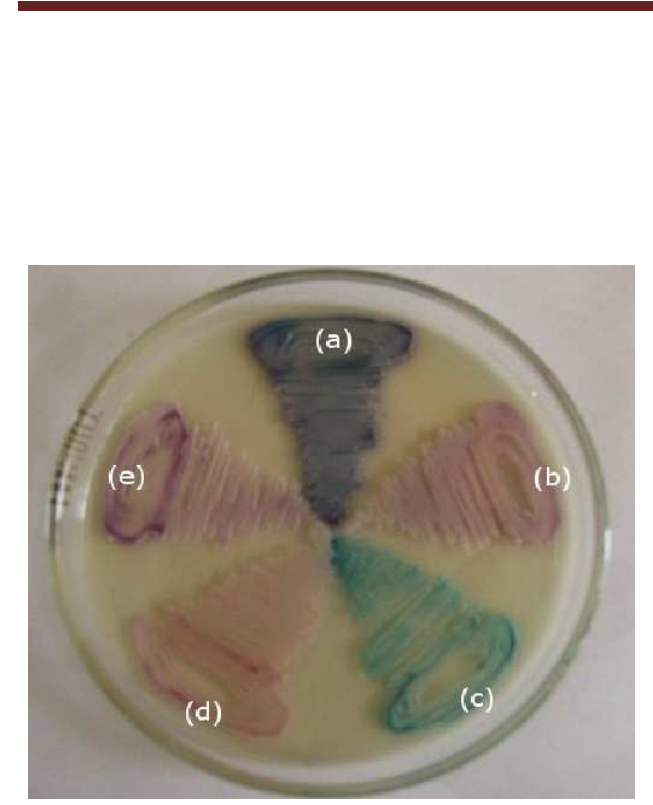

Figure 3.Colonies on CHROM agar : C.tropicalis (a), C.krusei (b), C.albicans (c), C.parapsilosis(d), and C.glabrata(e).

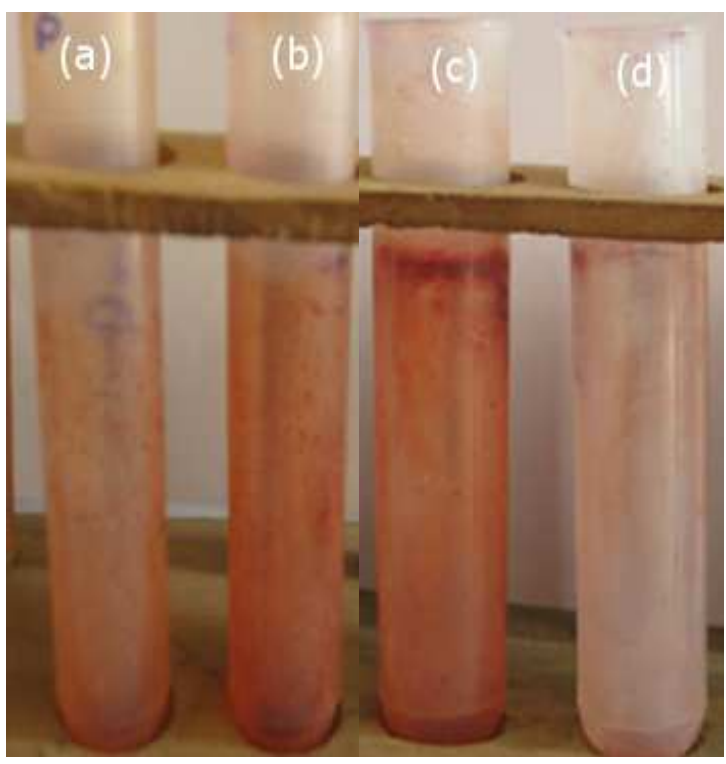

Figure 4.Biofilm positive 1+ (a), $2+(b), 3+(c)$ and biofilm negative(d)

\section{Chart 1}

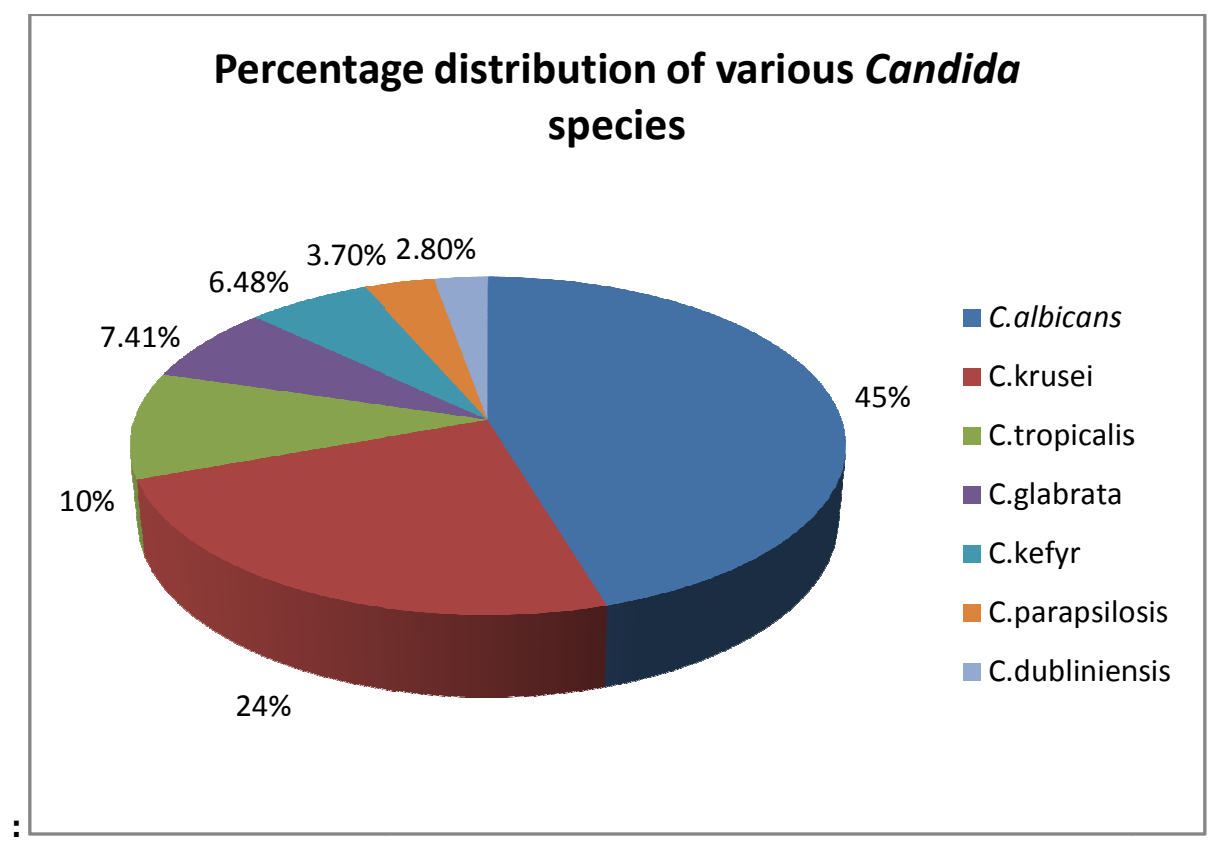




\section{Chart 2}

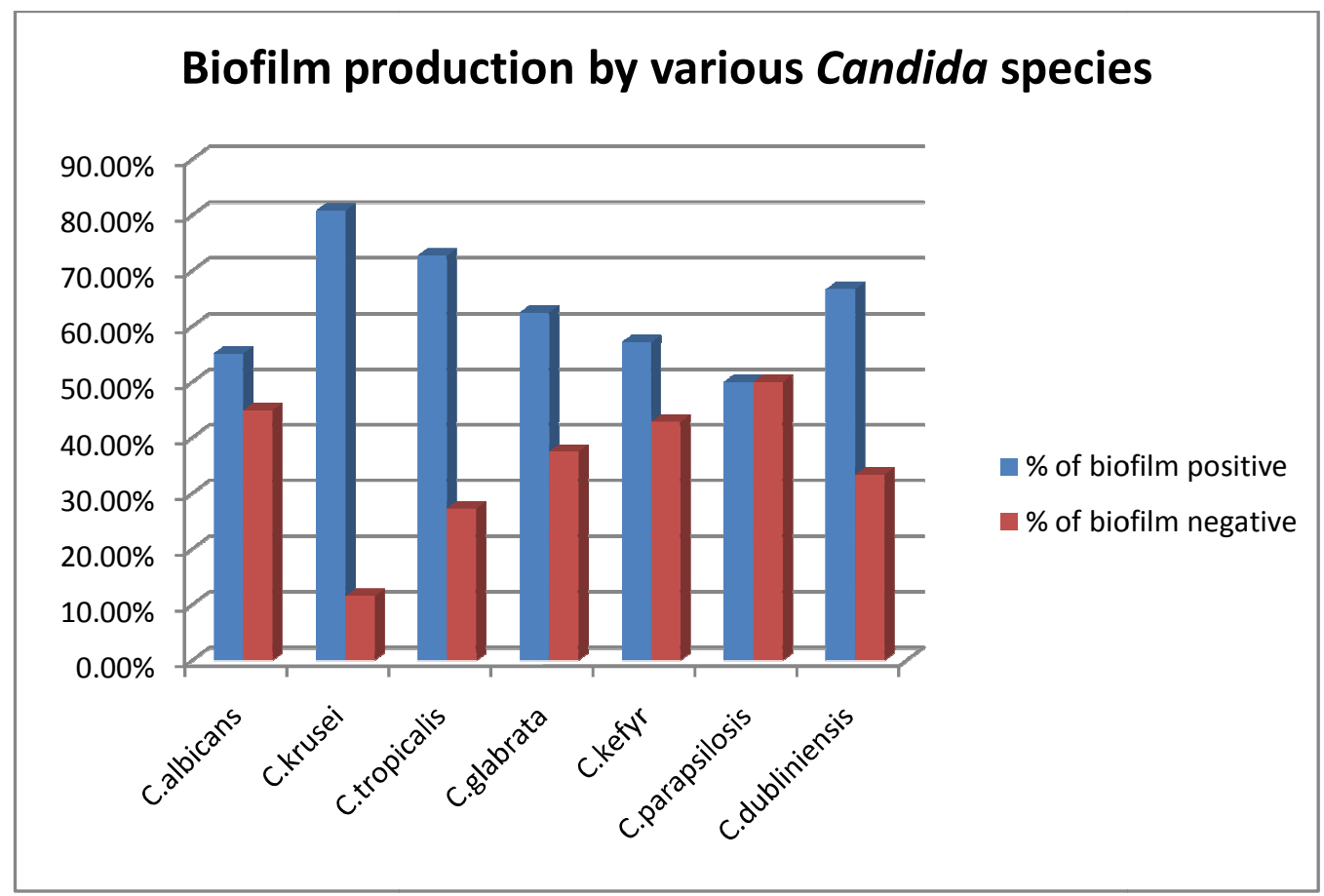

\section{Chart 3}

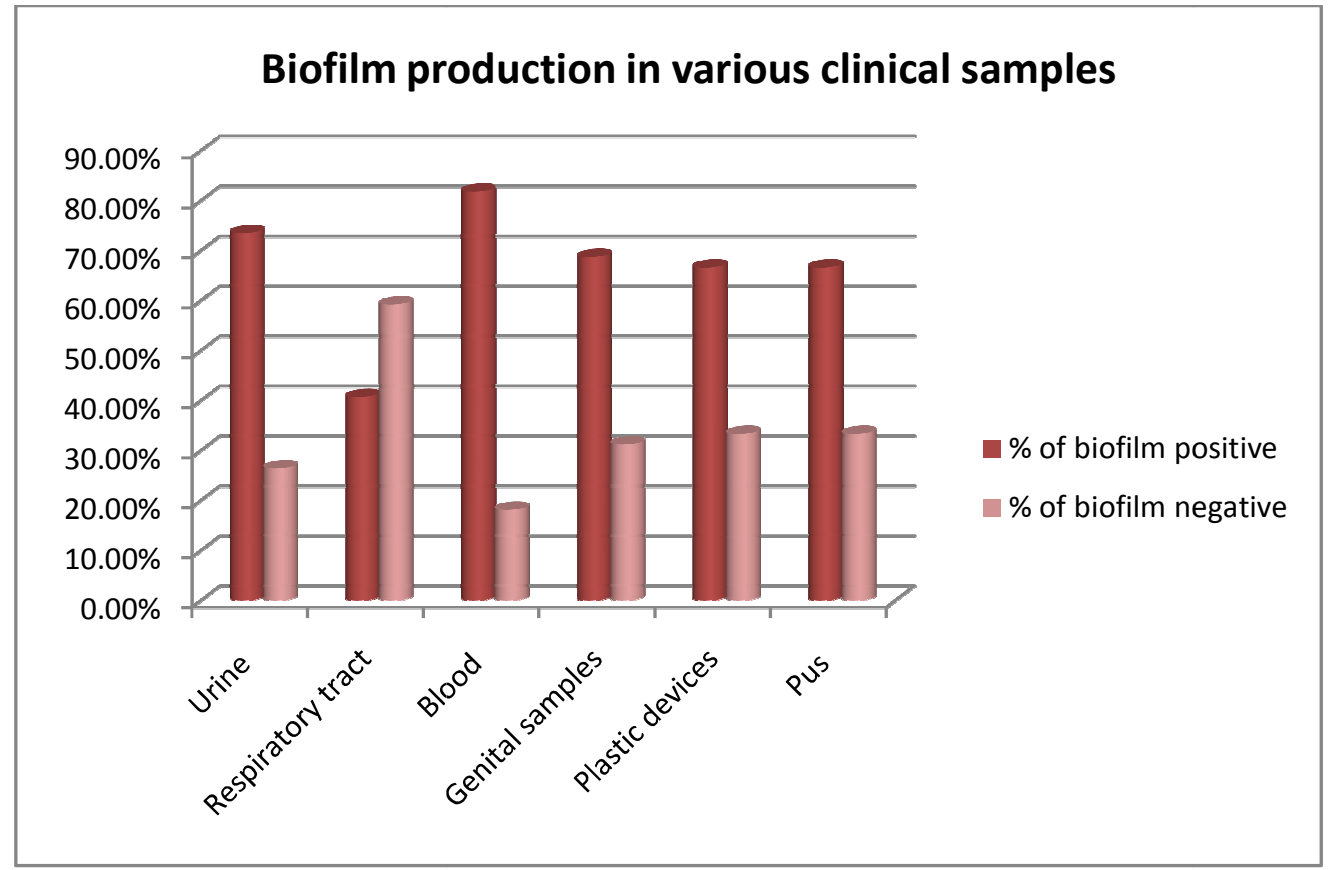

